\title{
Age of diagnosis of congenital hearing loss: Private v. public healthcare sector
}

\author{
I R T Butler, ${ }^{1}$ MB ChB, MMed (ORL); D Ceronio, ${ }^{2}$ BLog; T Swart, ${ }^{2}$ MCommPath (SLP \& Aud); G Joubert, ${ }^{3}$ BA, MSc \\ ${ }^{1}$ Department of Otorhinolaryngology, Faculty of Health Sciences, University of the Free State, Bloemfontein, South Africa \\ ${ }^{2}$ Audiologist in private practice, Bloemfontein, South Africa \\ ${ }^{3}$ Department of Biostatistics, Faculty of Health Sciences, University of the Free State, Bloemfontein, South Africa
}

Corresponding author: I Butler (butlerirt@ufs.ac.za)

\begin{abstract}
Background. The age of diagnosis of congenital hearing loss is one of the most important determinants of communication outcome. A previous study by the lead author had evaluated the performance of the public health services in Bloemfontein, South Africa (SA), in this regard. This study aimed to examine whether the private health services in the same city were any better.

Objective. To determine whether the age of diagnosis of congenital hearing loss (CHL) in children seen in the private healthcare sector in Bloemfontein, Free State Province, SA, was lower than that in the public healthcare system in the same city.

Methods. A comparative study design was utilised and a retrospective database review conducted. Data obtained from this study in the private healthcare sector were compared with data from a previous study in the public healthcare sector using the same study design.

Results. Forty-eight children aged $<6$ years with disabling hearing impairment (DHI) were identified in the private healthcare sector during the study period; $33 / 47(70.2 \%)$ did not undergo hearing screening at birth. The median age of diagnosis of DHI in the private healthcare sector was 2.24 years, and this was statistically significantly lower than the median age of diagnosis of 3.71 years in the public healthcare sector ( $p<0.0001 ; 95 \%$ confidence interval (CI) $0.99-2.0$ ). The median age of diagnosis of CHL in the private healthcare sector was 3.01 years in children who were not screened at birth, and 1.25 years in those who were screened at birth. This difference was statistically significant ( $p<0.01 ; 95 \%$ CI $0.72-2.47)$. We also compared the median age of diagnosis of CHL in children from the private healthcare sector who were not screened at birth (median 3.01 years) with that in children in the public healthcare sector (median 3.71 years). This difference was statistically significant $(p<0.01 ; 95 \%$ CI $0.41-1.56)$.

Conclusions. Children in the Free State are diagnosed with CHL at a younger age in the private healthcare sector than in the public healthcare sector. With the social and economic benefits of early intervention in cases of DHI well established internationally, SA healthcare providers in both the public and private sectors need to develop screening, diagnostic and (re)habilitation services for children with hearing impairment.
\end{abstract}

S Afr Med J 2015;105(11):927-929. DOI:10.7196/SAMJ.2015.v105i11.9576

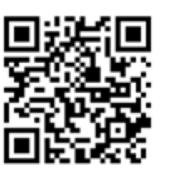

A recent study by Butler et al. ${ }^{[1]}$ found that the median age of diagnosis of congenital hearing loss (CHL) in a public sector tertiary referral hospital was 3.71 years. This is in sharp contrast to the Health Professions Council of South Africa (HPCSA) recommendation ${ }^{[2]}$ that diagnosis of hearing loss be achieved by 4 months of age (for clinic-based hearing screening programmes) and the international standard recommendation of 3 months of age (for hospital-based hearing screening programmes). One of the main reasons identified for this late age of diagnosis was the lack of newborn hearing screening programmes in the public health sector in South Africa (SA).

Universal newborn hearing screening (UNHS) is now a wellestablished practice in developed countries ${ }^{[2]}$ and has resulted in a significant lowering of the age of diagnosis of CHL, with consequent earlier intervention in managing the hearing impairment. The benefits of early intervention in cases of CHL are well established and include normalisation of communication skills and reduction of learning difficulties, cognitive developmental delays and the behavioural and emotional issues associated with untreated CHL. ${ }^{[3-11]}$

It has been estimated that $90 \%$ of SA children have no access to hearing screening services. ${ }^{[5]}$ This is mainly because UNHS is virtually non-existent in the public health service, which covers approximately $85 \%$ of the SA population; only $7.5 \%$ of public hospitals with birthing units provide some sort of hearing screening. ${ }^{[12]}$ Where hearing screening is available in the public sector, it is usually selectively applied to high-risk infants (such as those in neonatal intensive care units) or achieves screening in only a small percentage of the target population. The situation in the private healthcare sector is only marginally better. A 2011 publication found that only $53 \%$ of private hospitals offered some form of newborn hearing screening, and only $15 \%$ offered UNHS. UNHS is not covered by medical aid schemes as part of the 'birthing package.' As a result, hearing screening in the private healthcare setting represents an additional financial cost to parents, which many are unwilling to bear in view of their perceived low risk of CHL. ${ }^{[12]}$

Newborn hearing screening has been offered at the two major private hospitals in Bloemfontein, Free State Province, SA, since 2007. It is performed by audiologists from a single audiology practice and includes diagnostic tests on those children who do not pass the screening test. This provides an excellent opportunity to determine the age of diagnosis of $\mathrm{CHL}$ in the private healthcare setting and to compare this with the situation in the public healthcare setting.

\section{Objectives}

The primary aim of our study was to determine whether the age of diagnosis of CHL in children seen in the Deon Ceronio audiology practice in Bloemfontein (private healthcare sector) was lower than 
that in the public healthcare system in the same city. The hypothesis was that the age of diagnosis of CHL in the private healthcare setting would prove to be significantly lower than in the public healthcare setting.

Our secondary aims were to determine the age of first visit to the practice, and the time delay between first visit and diagnosis of hearing loss; to determine how many children underwent newborn hearing screening and what the influence of hearing screening was on the age of diagnosis of CHL; and to document any subsequent interventions.

\section{Methods}

A comparative study design was utilised and a retrospective database review conducted. Ethical approval was granted by the Ethics Committee of the Faculty of Health Sciences of the University of the Free State (ECUFS 140/2014), and written permission was obtained from the Deon Ceronio audiology practice to access the database. Data were compared with those generated from our previous study in the state healthcare sector, published in 2013. ${ }^{[1]}$

The inclusion criteria were as follows:

- Diagnosis of disabling hearing impairment (DHI) using the World Health Organization definition of DHI in children aged $<15$ years, i.e. pure-tone average in the better-hearing ear $<30 \mathrm{~dB}$

- Children aged $<6$ years at time of diagnosis

- Diagnosis during the period 1 January 2004 - 31 December 2013

- No history or findings on examination consistent with an acquired form of hearing loss.

Data were analysed and results summarised by frequencies and percentages (categorical variables) and means, standard deviations or percentiles (numerical variables). The private and public sector results were compared using $95 \%$ confidence intervals (CIs) for differences in means, medians or percentages.

\section{Results}

In the private sector, records were available for diagnostic hearing tests on 179 children aged $<6$ years at the time of testing during the 10 -year study period. Forty-eight children fulfilled the inclusion criteria of suffering from DHI. Twenty-six (54.2\%) were female and $22(45.8 \%)$ male. All had been referred to the practice with the suspicion of bilateral hearing loss.

Data pertaining to hearing screening at birth were available for 47 children. Thirty-three $(70.2 \%)$ of these 47 children did not undergo hearing screening at birth.

When the data were analysed (Table 1) our original hypothesis was proved correct, with a statistically significantly lower age of diagnosis of CHL in the private sector than in the public sector $(p<0.0001,95 \%$ CI 0.99 - 2.0).

The data from the private healthcare sector were further analysed according to different time periods (Table 2). This was performed in order to evaluate the influence of a newborn hearing screening programme (NHSP) on the age of diagnosis of CHL. The audiology practice initiated an NHSP in 2007 at both private hospitals in Bloemfontein. However, UNHS in these facilities was only widely accepted in 2009.

The period $2004-2008$ ('pre-screening') was compared with the period 2009 - 2013 ('post-screening'). The difference in age of diagnosis was not found to be statistically significant $(p=0.399,95 \%$ CI $-0.56-1.28$ ). This appears to be due to the small number of cases in each of the periods.

The median age of diagnosis of CHL in the private healthcare sector was found to be 3.01 years in children who were not screened at birth, and 1.25 years in those children who were screened at birth (Table 3). This difference was statistically significant ( $p<0.01 ; 95 \%$ CI 0.72 - 2.47). We also compared the median age of diagnosis of $\mathrm{CHL}$ in children from the private healthcare sector who were not screened at birth (median 3.01 years) with that in children in the public healthcare sector (median 3.71 years). This difference was statistically significant $(p<0.01 ; 95 \%$ CI $0.41-1.56)$.

The median age at first visit to the audiological practice was 2.13 years. The time delay between the first visit to the audiological practice and the diagnosis of CHL was a median of 13 days.

In terms of interventions, 15 children $(31.3 \%)$ eventually received cochlear implants and $21(43.8 \%)$ received conventional hearing aids with auditory-verbal/auditory-oral speech and language therapy. Ten children $(20.8 \%)$ received hearing aids but followed a total communication rehabilitation framework, and two (4.2\%) were referred for sign language-based rehabilitation.

\section{Discussion}

Many articles on hearing screening in SA have been published. ${ }^{[1,2,5,7,12]}$ All have dealt with the complexities of establishing NHSPs in our dichotomous healthcare system, and have emphasised the importance of early identification and intervention in cases of CHL.

To the best of our knowledge, this is the first SA study that has demonstrated a significant reduction in the age of diagnosis of $\mathrm{CHL}$ following implementation of an NHSP. The data (Table 3) showed a statistically significant difference in the age of diagnosis of DHI in children who underwent hearing screening at birth compared with children who did not. While the numbers were too small to demonstrate a statistical difference in the age of diagnosis when comparing the 'pre-screening period' (2004 - 2008) with the 'postscreening period' (2009 - 2013), the trend was clear, with median ages of 2.48 years and 1.57 years for each period, respectively.

While the age of diagnosis of CHL in children in the public sector (3.71 years) appeared comparable to that in children in the private sector who had not undergone hearing screening (3.01 years), this difference was statistically significant. This suggests that the implementation of UNHS is not the only factor determining the age of diagnosis of DHI in our population. Access to screening and

Table 1. Median age of diagnosis of $\mathrm{CHL}$ in the public and private healthcare sectors

\begin{tabular}{ll}
\hline Sector & Median age at diagnosis, years \\
\hline Public $(n=260)$ & 3.71 \\
Private $(n=48)$ & 2.24
\end{tabular}

Table 2. Median age of diagnosis of CHL when analysed according to time periods

\begin{tabular}{lll}
\hline Period & $\begin{array}{l}\text { Children identified } \\
\text { with DHI, } \boldsymbol{n}\end{array}$ & $\begin{array}{l}\text { Median age at } \\
\text { diagnosis, years }\end{array}$ \\
\hline $2004-2008$ & 19 & 2.48 \\
$2009-2013$ & 29 & 1.57
\end{tabular}

Table 3. Effect of newborn hearing screening in the private sector

\begin{tabular}{ll}
\hline Screened at birth? (private) & Median age at diagnosis, years \\
\hline No & 3.01 \\
Yes & 1.25
\end{tabular}


diagnostic audiology services once there is parental suspicion of hearing loss is of paramount importance, and delays in performance of the appropriate diagnostic testing are identified as being of great significance.

The median delay between the first visit to the audiology practice and the diagnosis of CHL in this study was 13 days. We did not compare this with the median delay between the first visit to the ENT clinic and the age of diagnosis in our study in the public sector (49 days), ${ }^{[1]}$ as our intention when examining this variable in the previous study had been to determine whether delays in our own hospital were responsible for the late age of diagnosis. However, since many of the children identified in the current study were referred by other audiologists for confirmation of the DHI, use of the age at first visit to the audiology practice' to calculate the delay to DHI was imprecise.

In terms of the interventions that took place, only two children (4.2\%) in the current study were referred for sign language-based intervention, while in the public sector 46 (26\%) children followed this path. This is a significant difference and confirms our contention that children are being identified too late in the public sector for spoken language-based interventions (hearing aids or cochlear implants). Fifteen children (31.3\%) in the current study received cochlear implants as part of their intervention.

National guidelines for early detection of hearing loss were published by the HPCSA in 2007. ${ }^{[2]}$ These guidelines proposed that CHL be diagnosed by 4 months of age, and that appropriate interventions be implemented by 8 months of age at the maximum. While the median age of diagnosis in the private healthcare sector (2.24 years) was found to be statistically significantly lower than the age of diagnosis in the public healthcare sector ( 3.71 years), this falls well short of the national guideline of 4 months. The implementation of the NHSP in the private healthcare sector resulted in a reduction of the age of DHI from 2.48 years to 1.57 years. However, $70.2 \%$ of the children with DHI in the current study did not undergo newborn hearing screening. The major reasons for the low percentage of screened infants are the novelty of the screening service during the early part of the study period, and the fact that the cost of this service is not covered by the 'birthing package' of the hospital and represents an additional cost that parents are unwilling to accept. More work needs to be done in sensitising parents to the importance of UNHS and advocating that medical aid schemes cover this cost.

This study has provided scientific evidence that newborn hearing screening is essential to lower the age of diagnosis of CHL and facilitate early intervention, the benefits of which have been shown internationally. ${ }^{[6,8-11,13]}$ Children who are identified and managed appropriately are likely to be able to join the mainstream school system. It is hoped that health administrators in the private and public sectors, medical insurance companies and practitioners will respond to this evidence. This is particularly important in our local context, given the dearth of schools that cater for children with hearing impairments.

\section{Conclusion}

This is the first SA study to demonstrate that implementation of UNHS reduces the age of diagnosis of CHL. There is a need to advocate for greater support from SA healthcare providers (both public and private) regarding development of screening, diagnostic and (re)habilitation services for children who are born with, or develop, hearing impairments.

\section{References}

1. Butler I, Basson S, Britz E, et al. Age of diagnosis of congenital hearing loss at Universitas Hospital, Bloemfontein. S Afr Med J 2013;103(7):474-475. [http://dx.doi.org/10.7196/SAMJ.6395]

2. Professional Board for Speech, Language and Hearing Professions, Health Professions Council of South Africa. Early Hearing Detection and Intervention Programmes in South Africa: Position Statement. Pretoria: HPCSA, 2007. www.hpcsa.co.za/Uploads/.../early_hearing_detection_statement. pdf (accessed 6 February 2012).

3. World Health Organization. Report of the Informal Working Group on Prevention of Deafness and Hearing Impairment Programme Planning. Geneva: WHO, 1991 (with adaptations from the Report of the First Informal Consultation on Future Programme Developments for the Prevention of Deafness and Hearing Impairment, WHO, Geneva, 23-24 January 1997, WHO/PDH/97.3).

4. Olusanya B. Highlights of the new WHO report on newborn and infant hearing screening and implications for developing countries. Int J Pediatr Otorhinolaryngol 2011;75(6):745-748. [http:// dx.doi.org/10.1016/j.ipporl.2011.01.036]

5. Swanepoel D, Storbeck C, Friedland P. Early hearing detection and intervention in South Africa. Int J Swanepoel D, Storbeck C, Friedland P. Early hearing detection and intervention in South Africa.

6. Yoshinaga-Itano C, Coulter D, Thomson V. Developmental outcomes of children with hearing los 6. Yoshinaga-Itano C, Coulter D, Thomson V. Developmental outcomes of children with hearing loss
born in Colorado hospitals with and without universal newborn hearing screening programs. Semin born in Colorado hospitals with and without universal newborn hearing

. Swanepoel D, Almec N. Maternal views on infant hearing loss and early intervention in a South African community. Int J Audiol 2008;47(Suppl. 1):S44-S48. [http://dx.doi.org/10.1080/14992020802252279] 8. Niparko J, Tobey E, Thal D, et al. Spoken language development in children following cochlear implantation. JAMA 2010;303(15):1498-1506. [http://dx.doi.org/10.1001/jama.2010.451]

9. Moog J, Geers A. Early educational placement and later language outcomes for children with cochlear implants. Otol Neurotol 2010;31(8):1315-1319. [http://dx.doi.org/10.1097/MAO.0b013e3181eb3226]

10. Geers A, Moog J, Biedenstein J, et al. Spoken language scores of children using cochlear implants compared to hearing age-mates at school entry. J Deaf Stud Deaf Educ 2009;14(3):371-385. [http:// dx.doi.org/10.1093/deafed/enn046]

11. Govaerts P, de Beukelaer C, Daemers K, et al. Outcome of cochlear implantation at different ages from 0 to 6 years. Otol Neurotol 2002;23(6):885-890. [http://dx.doi.org/10.1097/00129492-20021100000013

12. Meyer M, Swanepoel D. Newborn hearing screening in the private healthcare sector: A national study. Afr Med J 2011;101(9):665-667.

13. Colletti L, Mandala M, Shannon R, et al. Estimated net saving to society from cochlear implantation in infants: A preliminary analysis. Laryngoscope 2011;121(11):2455-2460. [http://dx.doi.org/10.1002/ lary.22131]

Accepted 5 October 2015. 